\title{
Preface
}

\section{Gender, Racial, and Socioeconomic Issues in Perioperative Medicine}

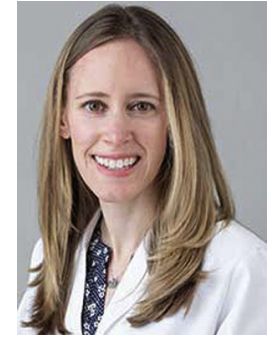

Katherine T. Forkin, MD

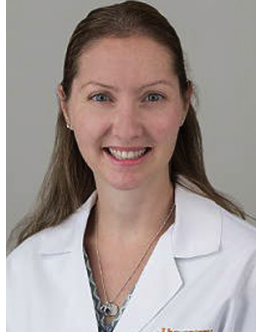

Lauren K. Dunn, MD, PhD Editors

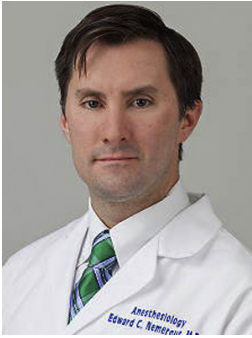

Edward C. Nemergut, MD

In diversity there is beauty and there is strength.

Gender, race, and socioeconomic status influence both patients and health care providers. These factors should be recognized, appreciated, and given weight when approaching patient care and considering the physician workforce. Although the influences of these factors may seem subtle, the effects on health care delivery and patients' outcomes can be profound. In this issue of Anesthesiology Clinics, we explore how gender, race, and socioeconomic status shape patients' experiences throughout the perioperative period.

Gender and racial differences may come into play preoperatively and lead to biases in preoperative testing. Furthermore, socioeconomic status may impact patients' access to and timely referral for care and influence how early in the course of their condition they present for surgical intervention. Awareness of the impact of these factors may help us to better prepare patients for surgery and ensure they are better optimized.

Many articles within this issue explore how gender and racial differences influence patient care during the perioperative period. For instance, racial disparities in cesarean section and labor analgesia deserve special attention. In addition, sex of the organ donor and recipient impacts solid organ transplantation in a variety of ways, from listing to graft survival and even mortality. We then focus on other unique patient populations. The article on transgender patients highlights the unique considerations throughout the perioperative experience that anesthesiologists should familiarize themselves with to provide improved care for these patients (eg, barriers to care, issues arising from hormone therapy). Race and culture also contribute to differences 
and disparities in care of pediatric patients, such as in the use of premedication, administration of opioid analgesia, and adultification of minority patients. These differences may be addressed through increased diversity of health care providers, affordable health care to improve access for economically disadvantaged groups, and rigorous data collection and reporting standards to identify disparity in patient care and outcomes.

The effect of race and gender on care and outcomes of the critically ill is also presented. In addition, gender differences following cardiac surgery, such as coronary artery bypass graft, valve replacement, and left-ventricular assist device surgeries, are detailed. Women have increased risk of morbidity and mortality and present with different preoperative risk profile compared with men presenting for cardiac surgery.

In the final articles, we focus on the effect of differences in gender and race in the workforce. The workforce is slowly changing to reflect the diversity of our patient population in the United States. Diversity in perioperative teams likely improves patient care, but more research is needed. Strategies for improving communication within perioperative teams are provided. Many challenges remain in academic anesthesiology for women and underrepresented racial minorities.

It is our hope that this issue of Anesthesiology Clinics will help readers appreciate the impact of patients' gender, race, and socioeconomic status on their perioperative experience, from preoperative evaluation through hospital discharge. We also anticipate readers will be able to take away from this issue the importance of race and gender in teamwork, communication, and career advancement of anesthesiologists. Finally, we would like to sincerely thank our authors for donating their time and energy to writing these articles.

Katherine T. Forkin, MD

Department of Anesthesiology University of Virginia Health System

PO Box 800710

Charlottesville, VA 22908-0710, USA

Lauren K. Dunn, MD, PhD

Department of Anesthesiology University of Virginia Health System

PO Box 800710

Charlottesville, VA 22908-0710, USA

Edward C. Nemergut, MD

Department of Anesthesiology University of Virginia Health System

PO Box 800710

Charlottesville, VA 22908-0710, USA

E-mail addresses:

ket2a@hscmail.mcc.virginia.edu (K.T. Forkin)

lak3r@hscmail.mcc.virginia.edu (L.K. Dunn) en3x@hscmail.mcc.virginia.edu (E.C. Nemergut) 